\title{
On the chemical potential of nanoparticle dispersion
}

\author{
H. Machrafi ${ }^{1,2}$ \\ ${ }^{1}$ Thermodynamics of Irreversible Phenomena, Institut de Physique, Université de Liège, Belgium \\ ${ }^{2}$ Physical Chemistry Group, Ecole Polytechnique, Université libre de Bruxelles, Belgium \\ *E-mail: h.machrafi@uliege.be
}

\begin{abstract}
Understanding of the mechanism describing the chemical potential of nanoparticle dispersions, whether from modelling or experimental perspectives, is missing in the literature. As nanofluids are widely used in engineering applications, predicting material properties correctly needs a correct formulation for their behaviour. Often, the chemical potential of mixing is used for such expressions. Although quite appropriate for polymer blends or binary solutions, it is not suitable for nanoparticle dispersions. This work proposes a new mechanism for the chemical potential of dispersions or suspensions from thermodynamic principles, relying on porous flow principles, proposing that it is the fluid that diffuses in between the nanoparticles. The proposed model is applied in the case of mass diffusion and the results compare well with molecular dynamics results and several experimental data, motivating the proposed mechanism for dispersions.
\end{abstract}

Keywords: chemical potential; nanoparticle dispersion; mass diffusion; nanofluid; thermodynamics; porous-like flow

\section{Introduction}

It should be emphasized that the chemical potential lies at the basics of properties such as the diffusion coefficient, the viscosity, the mass flux relaxation time and other properties of nanoparticle dispersions in fluids or liquid components. Often nanofluids are treated, with respect to their free energy of mixing, from which the chemical potential is obtained, as ideal or regular solutions in the lines of the Flory-Huggins theory [1-3]. Many suggestions have been offered to improve the expressions for the free energy of mixing and the corresponding chemical potential adapting the Flory-Huggins lattice model [4,5], but they have been performed for the purposes of polymer blends and binary mixtures properties, phase separation studies and diffusion in polymers [1-4,6-8]. Also, for some colloidal mixtures, the same model is used for the free energy $[9,10]$. However, these models are used in order to interpret experimental work and not to predict properties of colloidal mixtures. Furthermore, in such works it is generally assumed that all the components have the same type of entropic behaviour, following the statistical relation of the Boltzmann equation [4]. The latter means that colloidal mixtures are considered in those works as a mixture. That is just the issue. The question is whether nanoparticle or colloidal dispersions can be considered as solutions. In order to understand this, we should discuss what a dispersion is in practice and for what it is used. For many applications, nanoparticle dispersions, also called nanofluids [11,12], are used to enhance heat transfer for solar energy harvesting and storage, provide for smart cooling, or control the thermal management for electronics [12-17]. Nanoparticle dispersions are also used as a precursor for the preparation of nanocomposites or self-assembled structures in view of other applications, e.g. supercapacitors, batteries, electrodes or membranes [18-20]. The required effects depend heavily on the presence of the nanoparticles in the fluid, so that it is important 
that those nanoparticles are well dispersed. For that, they need to be well stabilized in order to avoid flocculation, settlement or agglomeration, which is quite a challenge [21]. This already indicates that nanoparticles are not so readily mixed in its base fluid, which the aforementioned standard mixing model would suggest, but that often its dispersion needs some preparation. Some dispersion mechanisms exist, such as electrostatic, steric, or electro-steric stabilization. These mechanisms rely on inter-particle repulsive forces (nanoparticle surface treatment by e.g. adsorption of ions, physical adsorption of charged species, accumulation or depletion of electrons), van der Waals inter-particle screening by macromolecular barriers attached to the nanoparticle surfaces and a combination of both, where surfactants or dispersing agents can also play a role [12]. These stabilization mechanisms suggest that the nanofluids are not really entropically mixed but rather dispersed. Indeed, it appears that the mixing entropy contribution from the nanoparticles is quite less than what the standard theory would propose [22,23]. This is also evidenced by observed incomplete mixing, due to an entropy decrease [24]. The incomplete mixing is due to the fact that the suspension or the dispersion is not a solution or mixture on a molecular basis, so that the nanoparticles do not have the same combinatorial occupation within the fluid as a solute would have on a molecular basis [9,23]. As preparations are needed in order to stabilize the suspension or dispersion, this gives rise to another phenomenon that influences the free energy of dispersion. The stabilization of the nanoparticles in the base fluid results into an enthalpic contribution to the free energy of dispersion that should compensate for the strongly reduced, maybe even to a negligible extent, entropic contribution of the nanoparticles. This enthalpic contribution stems from favourable, or attractive, interactions between the nanoparticle and the fluid (or matrix to be more general) as is evidenced from several modelling and experimental work $[22,23,25,26]$. At the same time, due to the interparticle repulsive forces, avoiding the nanoparticles to agglomerate, the nanoparticles movement is restricted with respect to that of the fluid (or matrix). This suggests that there is a restricted entropic contribution of the nanoparticles, that is being compensated by an enhanced enthalpic contribution for the dispersion to be stable. For predicting correctly physical properties of nanoparticle dispersions, one resorts often to the chemical potential, which implies that the free energy should take into account these observations, which are missed by the standard formulations of the free energy of mixing [27]. Although many studies have been performed on properties of nanofluids and on free energies of mixing, it is therefore rather surprising that no studies on the chemical potential of nanoparticles dispersion in fluids (or matrices) are performed, which necessitates new developments [27,28].

We propose therefore another way of looking at the free energy of dispersion of nanoparticles. The nanoparticles are considered to be stabilized in such a way that we can suggest a similarity with a porous-like model, where the fluid has liberty of movement and the nanoparticles have a fixed relative position to one another. Furthermore, in this model, the fluid undergoes a volume-pressure work going from a separated state to the state of dispersion. The nanoparticles, being assumed to have a fixed position to one another, do not cause a volume-pressure work. This means that in the proposed model, it is only the fluid that contributes to the entropy. The interaction between the nanoparticles and the fluid is modelled by means of an enthalpy of dispersion. We start with discussing the standard form for the free energy of mixing. As the density plays a role in not only porous flow but is also accounted for in some studies dealing with thermodynamics of dispersions [4,29-32], we propose a development for the free energy of mixing that takes into account density differences between the nanoparticles and the fluid or matrix. Subsequently, we apply the principles of our model in order to propose the free energy 
of dispersion. Since no studies on the chemical potential of nanoparticles dispersion has been performed, let alone experimental ones, our model is justified by other means. First, we consider an osmosis case and compare to another model based on kinetics. Finally, as one of the most direct uses of the chemical potential is related to the mass diffusion coefficient [8], we develop a model that predicts the mass diffusion of nanoparticle dispersions and compare it with molecular dynamics models and experiments.

\section{Theory: free energy of an ideal solution}

\subsection{Standard formulation}

We discussed on whether to consider the dispersions as binary solutions or not. Although we argued, substantiated by several other works, that nanoparticle dispersions are generally not to be seen as solutions, it is, in order to appreciate the difference, interesting to discuss the free energy of mixing of an ideal solution. Often, for an ideal solution, the so-called lattice model is used, where each lattice point consists of a particle of a component regardless of its size and composition. Considering that each lattice point has the same volume $v_{0}$, the number of combinations each component can fill such a lattice point, taking into account the presence or absence of the other component, leads to the mixture entropy $\Delta S$ (change from a reference value) for each component. The volume of such a lattice point is arbitrary and, if used consequently, should not influence the outcome. For further purposes, it is then convenient to choose such a lattice volume equal to the volume of a nanoparticle $V_{p}$. For an ideal (index $i d$ ) mixture (index $m$ ), the mixture enthalpie change $\Delta H$ is zero, so that the free Gibbs energy change $\Delta G$ is given by $\Delta G_{m, i d}=\Delta H_{m, i d}-T \Delta S_{m, i d}=-T \Delta S_{m, i d}$. If we have $N$ numbers of lattice sites, containing $N_{f}$ number of base fluid molecules (each one small enough to occupy a lattice site) and $N_{p}$ number of nanoparticles (assumed to occupy also one lattice site, the nanoparticles assumed to be sufficiently small to be regarded as a point mass), the total number of microstates would equal

$W=\frac{N !}{N_{p} ! N_{f} !}$

Boltzmann's equation of entropy would give

$S_{p}=k_{B} \ln (W)=k_{B} \ln \left(\frac{N !}{N_{p} ! N_{f} !}\right)$

For a pure fluid, only one undistinguishable combination exists, i.e. $S_{f}=k_{B} \ln (1)=0$. The total number of particles equals $N=N_{p}+N_{f}$ and the total volume equals $V_{t}=N_{p} V_{p}+N_{f} V_{f}=$ $N_{p} V_{p}+N_{f} V_{p}$, so that $\varphi=\frac{N_{p}}{N_{p}+N_{f}}$. Defining $\Delta S_{m, i d}=S_{p}-S_{f}$, and using Stirling's approximation for the logarithm of factorials, gives

$\Delta S_{m, i d}=-k_{B}\left(N_{p} \ln (\varphi)+N_{f} \ln (1-\varphi)\right)$

For a regular solution (subscript $r$ ), the particle-particle, solvent-solvent and particle-solvent interactions are to be taken into account as well. We can then speak of the difference in interaction energy $\Delta w$ between like and unlike neighbours:

$\Delta \omega=\varepsilon_{p s}-\frac{1}{2}\left(\varepsilon_{p p}+\varepsilon_{s s}\right)$ 
In this case, the enthalpy of mixing is non-zero, i.e. $\Delta G_{m, r}=\Delta H_{m, r}-T \Delta S_{m, i d}$. It can be understood as that each interaction between a particle and the solvent goes at the expense of the average of the other two interactions. If $z$ is the coordination number standing for the number of nearest lattice neighbours for both the solvent and particles, then $z N_{p}$ is the number of nearest-neighbour sites to all the particles. Multiplying this by the probability $\frac{N_{f}}{N_{p}+N_{f}}$ that any such site is occupied by a fluid molecule, we obtain the total number of particle-solvent interactions $z N_{p} \frac{N_{f}}{N_{p}+N_{f}}=z N_{p}(1-\varphi)$. The enthalpy of mixing is then equal to

$\Delta H_{m, r}=z N_{p}(1-\varphi) \Delta \omega$

A nanoparticle-solvent interaction parameter $\left(\xi_{p f}\right)$ is defined in analogy to the polymer-solvent parameter as

$\xi_{p f} \equiv-2 \chi_{p f}=-2 \frac{z \Delta \omega}{k_{B} T}$

where $\chi_{p f}$ is the well-known Flory-Huggins interaction parameter, generally also used for nanoparticle suspensions, $[7,33]$ so that the enthalpy of mixing becomes

$\Delta H_{m, r}=-k_{B} T \frac{\xi_{p f}}{2} N_{p}(1-\varphi)$

The minus sign in the expression of the $\xi_{p f}$ parameter indicates that for $\xi_{p f}>0$ (the $\chi_{p f}$ parameter is then negative) an exothermic enthalpy of mixing is obtained, which favours the stability of a nanofluid dispersion and guarantees $\Delta G_{m, r}<0$ [33]. Finally, we obtain the Gibbs free energy per unit mass for a regular solution to be

$\Delta G_{m, r}=k_{B} T\left(N_{p} \ln (\varphi)+N_{f} \ln (1-\varphi)-\frac{\xi_{p f}}{2} N_{p}(1-\varphi)\right)$

Dividing by the total volume of the system (assuming that each volume package is equivalent to the volume of a nanoparticle, i.e. $V_{f}=V_{p}$ ) leads to the free energy of mixing per unit volume of

$\Delta G_{m, r}=\frac{k_{B} T}{V_{p}}\left(\varphi \ln (\varphi)+(1-\varphi) \ln (1-\varphi)-\frac{\xi_{p f}}{2} \varphi(1-\varphi)\right)$

which resembles the well-known Flory-Huggins model for the free energy of mixing in a regular solution [1-3]. However, the effect of mass transport is not taken into account here. For this, we should consider the effect of the material densities.

\subsection{Effect of material densities}

Imagine there is a diluted solution that behaves like a gas (in the way one would do for Brownian particles). This solution contains a solute (regarded equivalent to the nanoparticles for the purposes of the present discussion) with pressure $p_{p}$ and specific volume $\frac{1}{\rho_{p}}$ and a solvent 
(the base fluid or matrix) with pressure $p_{f}$ and specific volume $\frac{1}{\rho_{f}}$. Here $\rho_{p}$ and $\rho_{f}$ are the mass densities of the particles and the fluid, respectively. Combining the First and Second Law of Thermodynamics, we have that the internal energy $d u$ per unit mass, for a fixed composition of the solution, changes by

$d u=T d s-d w$

where $d w$ is the change in work per unit mass. Let us now imagine that in a reversible process, the solute and solvent are slowly compressed each one of them in a different part within the system, separated by an impermeable membrane. Moreover, in case of an ideal solution, the internal energy does not change during this compression, so that $d u=0$. In a reverse process, taking that imaginary membrane away, the solute and solvent expand to equilibrium, resulting spontaneously into an ideal mixture. The latter process generates an entropy of mixing per unit mass for an ideal solution. This image is given schematically in Fig. 1.

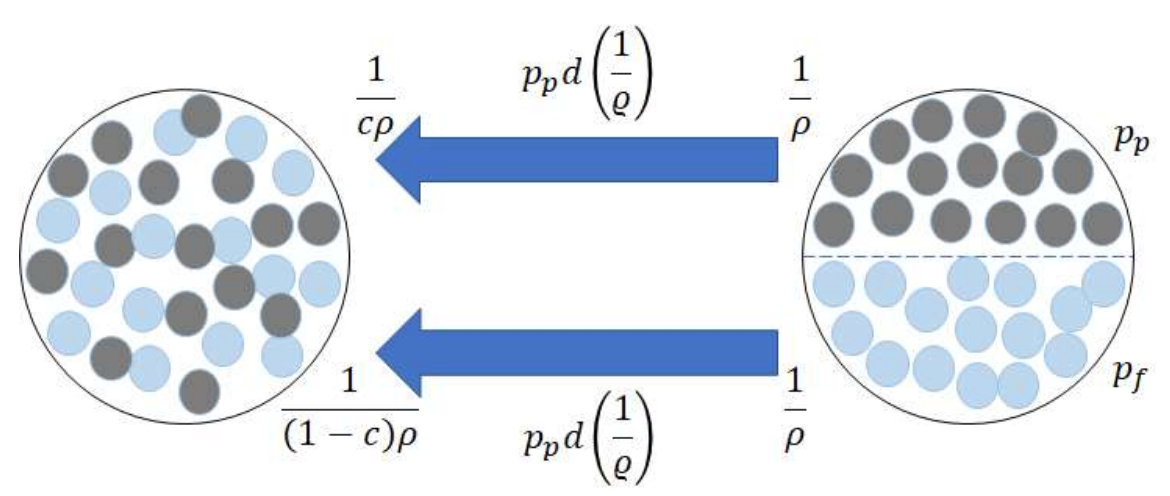

Figure 1: Schematic representation of the specific-volume-pressure work generating entropy of mixing for an ideal solution

During this ideal mixing, the work can be expressed by a specific volume-pressure work

$d w=\left.p_{p} d \frac{1}{\varrho}\right|_{f}+\left.p_{f} d \frac{1}{\varrho}\right|_{p}$

with the specific volume $\frac{1}{\varrho}$ (defined as the total volume a component occupies in a certain control volume divided by the mass of that component) as variable and $p=p_{p}+p_{f}$ is the total pressure, assumed to be constant. The indices $f$ and $p$ of the vertical bars in Eq. (11) stand for keeping the value of the corresponding variable for, respectively, the fluid and the particle constant at derivation. As the changes in the volume-pressure work are per unit mass, we deal here with 'mass clusters' of solutes and solvents with different densities and volumes (contrary to the lattice model, discussed previously) i.e. $m_{f} \equiv m_{p}$. The mass fraction is defined here as $c=\frac{N_{p} m_{p}}{N_{p} m_{p}+N_{f} m_{f}}$, with $m_{t}=N_{p} m_{p}+N_{f} m_{f}$ is the total mass. In the present model, the pressure of the solute and solvent can be given as $p_{p} V=N_{p} k_{B} T$ and $p_{f} V=N_{f} k_{B} T$, respectively. Per unit mass, we can write the following state equations:

$p_{p} \frac{1}{\varrho}=\frac{N_{p}}{m} k_{B} T$ 
$p_{f} \frac{1}{\varrho}=\frac{N_{f}}{m} k_{B} T$

Filling this in Eqs. (10)-(11) leads to

$$
T d s=k_{B} T\left(\frac{N_{p}}{m_{t}}\left(\frac{d\left(\frac{1}{\varrho}\right)}{\left(\frac{1}{\varrho}\right)}\right)_{f}+\frac{N_{f}}{m_{t}}\left(\frac{d\left(\frac{1}{\varrho}\right)}{\left(\frac{1}{\varrho}\right)}\right)_{p}\right)
$$

Integrating $d s$ in Eq. (14) from a state of separation to a state of full mixing gives simply the entropy of mixing per unit mass $\Delta s_{m, i d}$. We can observe that for the nanoparticles the variable $\frac{1}{\varrho}$ is initially given by the total volume of nanoparticles divided by the total mass of the nanoparticles in the separated compartment, which corresponds to the total volume divided by the total mass in the separated compartment, i.e. $\frac{1}{\rho}$. In state of mixing the variable $\frac{1}{\varrho}$ becomes the total volume of nanoparticles divided by the total mass of nanoparticles, the latter of which corresponds to the mass fraction of nanoparticles multiplied by the total mass in the mixed state, i.e. $\frac{1}{c \rho}$. The mass fraction is defined as $c=\frac{\varphi \rho_{p}}{\rho}=\frac{\varphi \rho_{p}}{\varphi \rho_{p}+(1-\varphi) \rho_{f}}$. For the base fluid, we can make the same reasoning and conclude that the variable $\frac{1}{\varrho}$ goes from $\frac{1}{\rho}$ to $\frac{1}{(1-c) \rho}$. Furthermore, we can notice from the previous definitions that $\frac{N_{p}}{m_{t}}=\frac{c}{m_{p}}$ and $\frac{N_{f}}{m_{t}}=\frac{1-c}{m_{f}}=\frac{1-c}{m_{p}}$ with $m_{p}=\rho_{p} V_{p}$ and $m_{f}=\rho_{f} V_{f}$. The integration of Eq. (14) leads finally to

$\Delta s_{m, i d}=-\frac{k_{B}}{\rho_{p} V_{p}}(\ln (c)+(1-c) \ln (1-c))$

For a regular solution, we remind that $z N_{p}$ is the number of nearest-neighbour sites to all the particles. As we proposed for the entropy, we suggest that also the interaction energy depends on the density of the particle and fluid clusters. We can say that two clusters with the same volume and a different mass affect each other's interaction per unit mass in a different way. Therefore, per unit mass of the system, the probability that a particle's interaction is affected by a neighbouring site should be mass-averaged. As such, the probability that a particle's interaction per unit mass is affected by a neighbouring fluid cluster is $\frac{N_{f} m_{f}}{N_{p} m_{p}+N_{f} m_{f}}=1-c$. The total number of particle-solvent interactions per unit mass is then $z N_{p}(1-c)$. The enthalpy of mixing per unit mass equals

$\Delta h_{m, r}=\frac{\Delta H_{m, r}}{m_{t}}=z \frac{c}{\rho_{p} V_{p}}(1-c) \Delta \omega$

Such a mass-fraction dependence for the enthalpy of mixing is similar to what is used for studying state transitions in polymer blends [30,34,35]. Defining again $\xi_{p f} \equiv-2 \frac{z \Delta \omega}{k_{B} T}$, the enthalpy of mixing per unit mass becomes

$\Delta h_{m, r}=-k_{B} T \frac{\xi_{p f}}{2} c(1-c)$ 
Finally, we obtain the Gibbs free energy of mixing per unit mass for a regular solution to be

$\Delta g_{m, r}=\frac{k_{B} T}{\rho_{p} V_{p}}\left(c \ln (c)+(1-c) \ln (1-c)-\frac{\xi_{p f}}{2} c(1-c)\right)$

The discussion lead between Eqs. (7) and (8) apply here as well, observing that, keeping in mind $\xi_{p f}>0$, the sign of $\Delta h_{m, r}$ is negative as well, so that $\xi_{p f}>0$ favours the stability of a nanoparticle dispersion and guarantees $\Delta g_{m, r}<0$.

The entropy of mixing is argued to be valid for an ideal solution of a solute with mixing on atomic level. Indeed, often the free energy of mixing is shortened as $\Delta g_{m, i d}=\frac{k_{B} T}{\rho_{p} V_{p}} \operatorname{cln}(c)$ for an ideal solution, resulting into the well-known chemical potential for strongly diluted solutions $(c \ll 1): \eta_{i d}=\eta_{p}=\frac{k_{B} T}{\rho_{p} V_{p}}(1+\ln (c))$, which would give $\frac{\partial \eta_{i d}}{\partial c}=\frac{k_{B} T}{\rho_{p} V_{p}} \frac{1}{c}$, similar to the result in [8]. Beyond the dilute-limit, the chemical potential for polymer blends or binary liquid solutions can be argued to be reasonably given by $\eta_{i d}=\frac{k_{B} T}{\rho_{p} V_{p}} \ln \left(\frac{c}{1-c}\right)$, with $\frac{\partial \eta_{i d}}{\partial c}=\frac{k_{B} T}{\rho_{p} V_{p}} \frac{1}{c(1-c)}$.

As mentioned earlier, this is not correct for nanoparticle dispersions and a new development is proposed. We can easily see that for $\rho=\rho_{f}=\rho_{p}$, Eq. (9) is found back by multiplying with the density, which shows that the present thermodynamic representation leads to the same result as the kinetic one. This encourages the use of such a thermodynamic representation for proposing a new development applicable to nanoparticle dispersions.

\section{Chemical potential of dispersion}

At constant temperature and pressure and in absence of a chemical reaction, a change of the Gibbs free energy per unit mass for a regular solution is given by

$d g=\eta_{1} d c_{1}+\eta_{2} d c_{2}=\eta_{1} d c_{1}-\eta_{2} d c_{1}=\eta d c$

recalling that $d c_{2}=-d c_{1}$ and that we define $c_{1} \equiv c$. As such, the chemical potential difference, hereafter simply called the chemical potential, is defined as

$\eta=\left(\frac{d g}{d c}\right)_{T, p, N}$

where the subscripts $T, p$ and $N$ denote a constant temperature, pressure and mass fraction of the other components. Stabilization of nanoparticle dispersions, due to the various aforementioned mechanisms (including also dispersing agents or surfactants), causes an entropy decrease, suggesting that the overall entropic contribution due to the nanoparticles could be neglected [22-24]. This motivates the use of the porous-like model in this work. Let us imagine that the nanoparticles, although allowed to flow simultaneously in one direction, keep a constant position with respect to one another. The fluid, however, is free to move between the nanoparticles as if the latter forms a porous medium. This image is schematized in Fig. 2. 


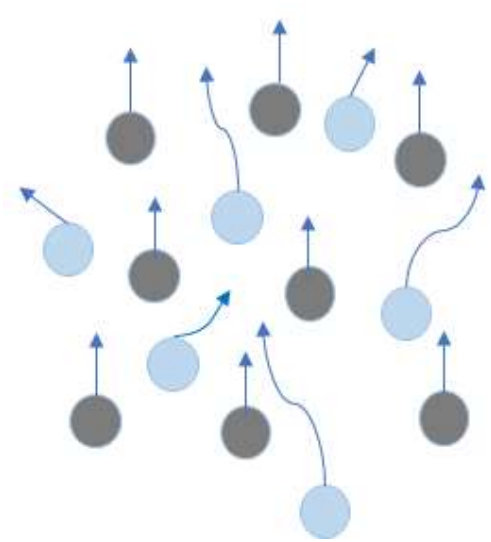

Figure 2: A scheme showing the nanoparticles going in a direction as if they constituted a porous medium going at a constant speed not changing positions to one another, while the fluid travels freely in between them

Let us imagine a control volume compartment that contains a certain volume of the dispersion, which is then slowly and reversibly separated into two compartments, where the nanoparticles are placed into a compartment with the same volume, while the fluids are compressed. Since this process is taken as reversible, we now allow the nanoparticles to be moved to the compartment of the dispersion, for which the volume does not change, which means that per unit mass of nanoparticles, the total volume remains constant, i.e. there is no change in the specific volume. At the same time, the fluid is allowed to expand in between the nanoparticles, which causes a change in the volume of the fluid per unit mass of fluid and thereby a specificvolume-pressure work. This means that the entropic contribution to the free energy, in the present model, comes from the specific-volume-pressure work caused by the fluid. This process is shown in Fig. 3.

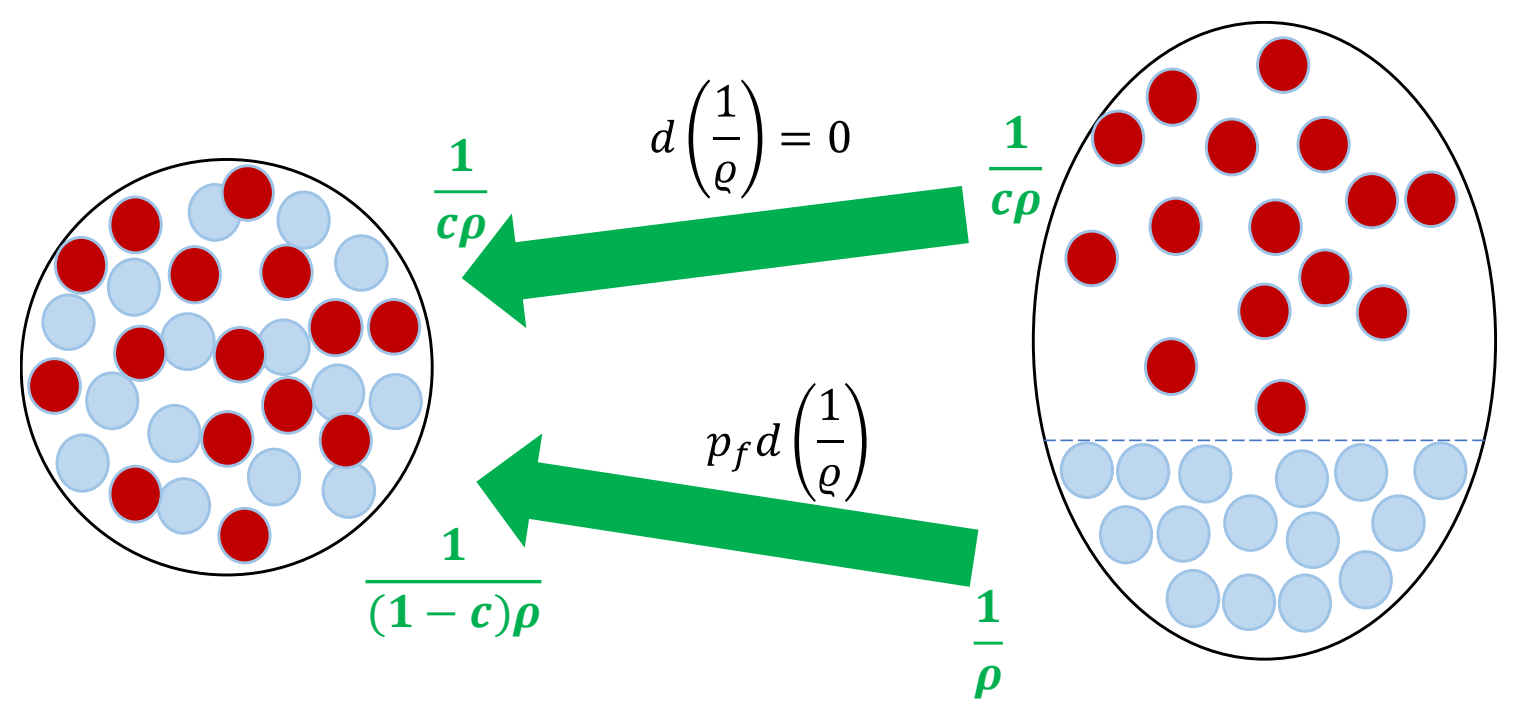

Figure 3: Schematic representation of the specific-volume-pressure work generating entropy of mixing for an ideal dispersion as proposed by our model

This process is thermodynamically described by using Eqs. (10)-(11), but in this case we have that the specific-volume-pressure work due to the nanoparticles is negligible, so that we take $p_{p} d\left(\frac{1}{\varrho}\right)=0$ and $d w=p_{f} d\left(\frac{1}{\varrho}\right)$. We can then write for the entropy 
$T d s=k_{B} T \frac{N_{f}}{m}\left(\frac{d\left(\frac{1}{\varrho}\right)}{\left(\frac{1}{\varrho}\right)}\right)$

Integrating, where $\frac{1}{\varrho}$ goes from $\frac{1}{\rho}$ to $\frac{1}{(1-c) \rho}$, reminding that in our model we defined $\frac{N_{f}}{m}=\frac{1-c}{\rho_{f} V_{f}} \equiv$ $\frac{1-c}{\rho_{p} V_{p}}$, gives

$\Delta s_{i d}=k_{B} \frac{1-c}{\rho_{p} V_{p}} \ln \left(\frac{1}{1-c}\right)$

For an ideal solution, the Gibbs free energy for ideal nanoparticle dispersion is then easily obtained as

$\Delta g_{i d}=\frac{k_{B} T}{\rho_{p} V_{p}}(1-c) \ln (1-c)$

For a regular solution, the enthalpy of mixing, Eq. (17), should be added to Eq. (23). The nondimensional free energy for an ideal dispersion, $\Delta g_{i d}^{n}=\Delta g_{i d}\left(\frac{k_{B} T}{\rho_{p} V_{p}}\right)^{-1}$, is shown in Fig. 4. For comparison, the same is shown for the free energy of mixing of an ideal solution $\left(\xi_{p f} \equiv 0\right)$ from Eq. (18).

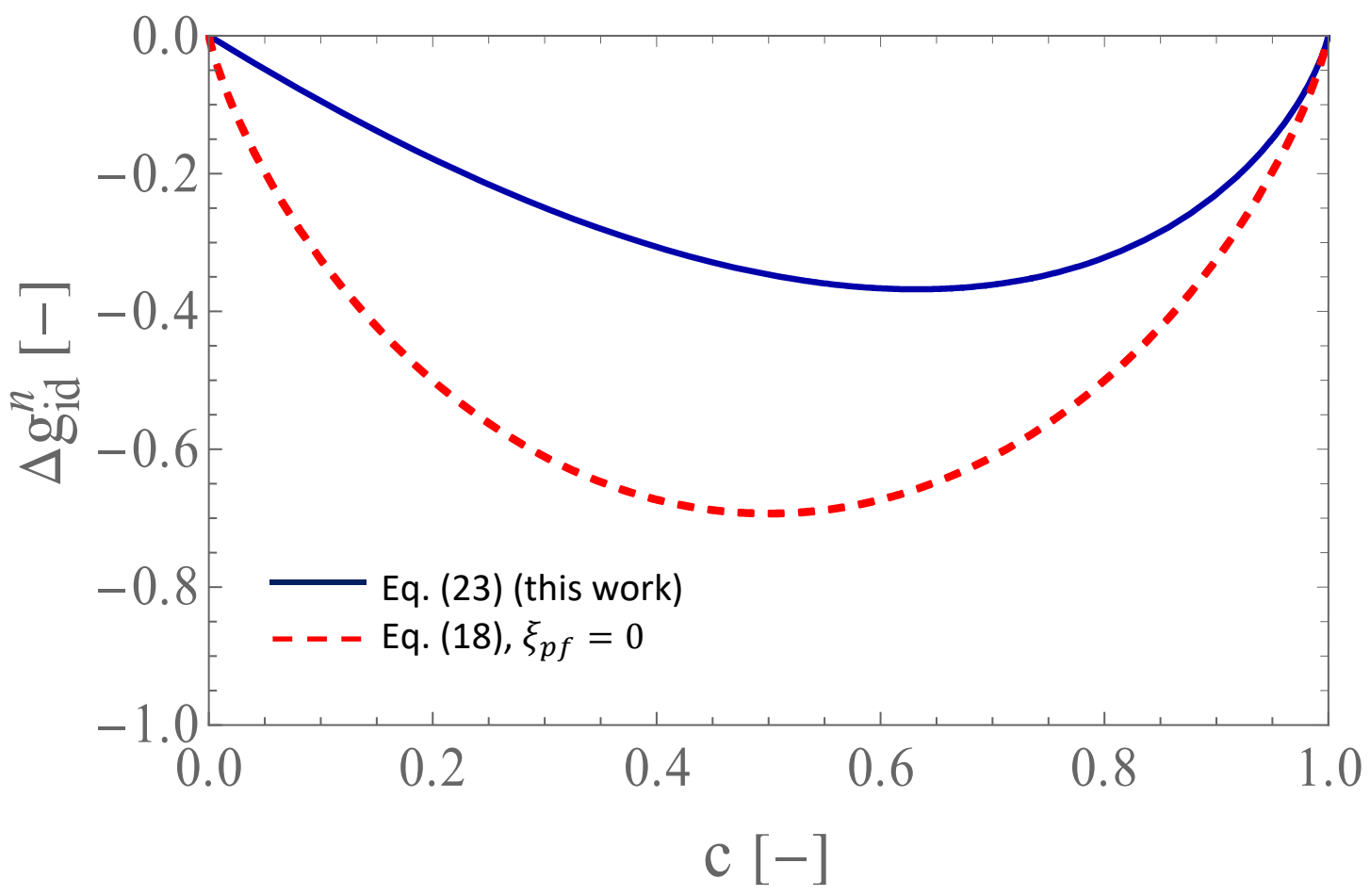

Figure 4: Non-dimensional free energy for ideal nanoparticle dispersion $\Delta g_{i d}^{n}$ as a function of nanoparticle mass fraction $c$ for nanoparticle dispersions, Eq. (23) and ideal solutions, Eq. (18).

\section{Results and Discussion}

\subsection{Osmosis}


In a kinetic explanation for the entropy of mixing [36], it is proposed that there exists an equivalence between the mechanisms responsible for diffusive osmosis and the free energy of mixing in case of an ideal solution. Proposing a kinetic solution-blocking model, they suggest that the Boltzmann factor $\varepsilon=e^{-d G / k_{B} T}$ should be equal to the molar fraction of the fluid in the solution. The osmosis would then be responsible for the mixing entropy [36,37]. Using variables per unit mass, it would mean that the Boltzmann factor $\varepsilon=e^{-d g / k_{B} T}$ is equal to the mass fraction of the fluid. It is then easy to verify that the osmotic pressure for such an osmosis case is proportional to the natural logarithm of the fluid concentration [36]. It is interesting to use our model to verify such an equivalence. For this, we first take the chemical potential of the fluid

$\eta_{f}=\frac{\partial \Delta g}{\partial(1-c)}=-\frac{\partial \Delta g}{\partial c}=\frac{k_{B} T}{\rho_{p} V_{p}}(1+\ln (1-c))=\eta_{f, 0}+\frac{k_{B} T}{\rho_{p} V_{p}} \ln (1-c)$

with $\eta_{f, 0} \equiv \frac{k_{B} T}{\rho_{p} V_{p}}$. In analogy with $[36,38]$, but taking into account density differences, the osmotic pressure for an osmosis setup is defined as $\Pi=-\rho\left(\eta_{f}-\eta_{f, 0}\right)$. With Eq. (24), this leads to

$\Pi=-k_{B} T \frac{\rho}{\rho_{p} V_{p}} \ln (1-c)$

For a strongly diluted dispersion, we can make the assumption that $\ln (1-c) \approx-c$. Eq. (25) becomes

$\Pi=k_{B} T \frac{\rho}{\rho_{p} V_{p}} c=\frac{k_{B} T}{m_{p}}[c]$

with $[c]$ the mass concentration of nanoparticles. Eq. (26) can also be written as

$\Pi=\frac{k_{B} T}{m_{p}}[c]=k_{B} T \frac{n_{p}}{V} \approx R T[M]$,

with $[M]$ the molar concentration, which corresponds interestingly to Van't Hoff's equation. We can observe that the same result for the osmotic pressure is obtained as in [36]. This also means that our porous-like model gives the same results as the solute-blocking model in what concerns the osmotic pressure, although departing from different principles. Moreover, a similar deduction is also performed by [39,40], motivating the use of the free energy of dispersion in Eq. (23).

\subsection{Behaviour of the free energy of dispersion}

Reflecting on the previous discussions and recalling that the initial model that we proposed to work on is fluid flow through a porous-like structure, we can say the following. Whether it is assumed that the nanoparticle contribution to the increase of the entropy of mixing is cancelled out by a decrease of entropy due to the stabilization of the dispersion, or whether its contribution can be neglected due to the heterogeneous character of the dispersion or whether a kind of osmotic-pressure model can be proposed, all seem to suggest it is reasonable to use Eq. (23) for the ideal free energy of nanoparticle dispersion. Adding finally the contribution of the enthalpy of mixing, Eq. (17), the following Gibbs free energy for nanoparticle dispersions is proposed: 
$\Delta g_{m, n p}=\frac{k_{B} T}{\rho_{p} V_{p}}\left((1-c) \ln (1-c)-\frac{\xi_{p f}}{2} c(1-c)\right)$

As mentioned in the discussion between Eqs. (7) and (8), for $\xi_{p f}>0$, it can be easily seen that $\Delta g_{m, n p}<0$, which is a criterion for nanofluid dispersion stability. Without the effect of the dispersing agents, the free energy would most probably be closer to Eq. (18). It is interesting to show the qualitative effect of $\xi_{p f}$ (recall that in this paper $\xi_{p f}>0$ and $\xi_{p f}<0$ stand for particle-fluid attraction and repulsion, respectively) on the free energy and for this purpose the non-dimensional free energy $\Delta g_{m, n p}^{n}=\Delta g_{m, n p}\left(\frac{k_{B} T}{\rho_{p} V_{p}}\right)^{-1}$ from Eq. (28) is drawn in Fig. 5 as a function of the nanoparticle mass fraction.

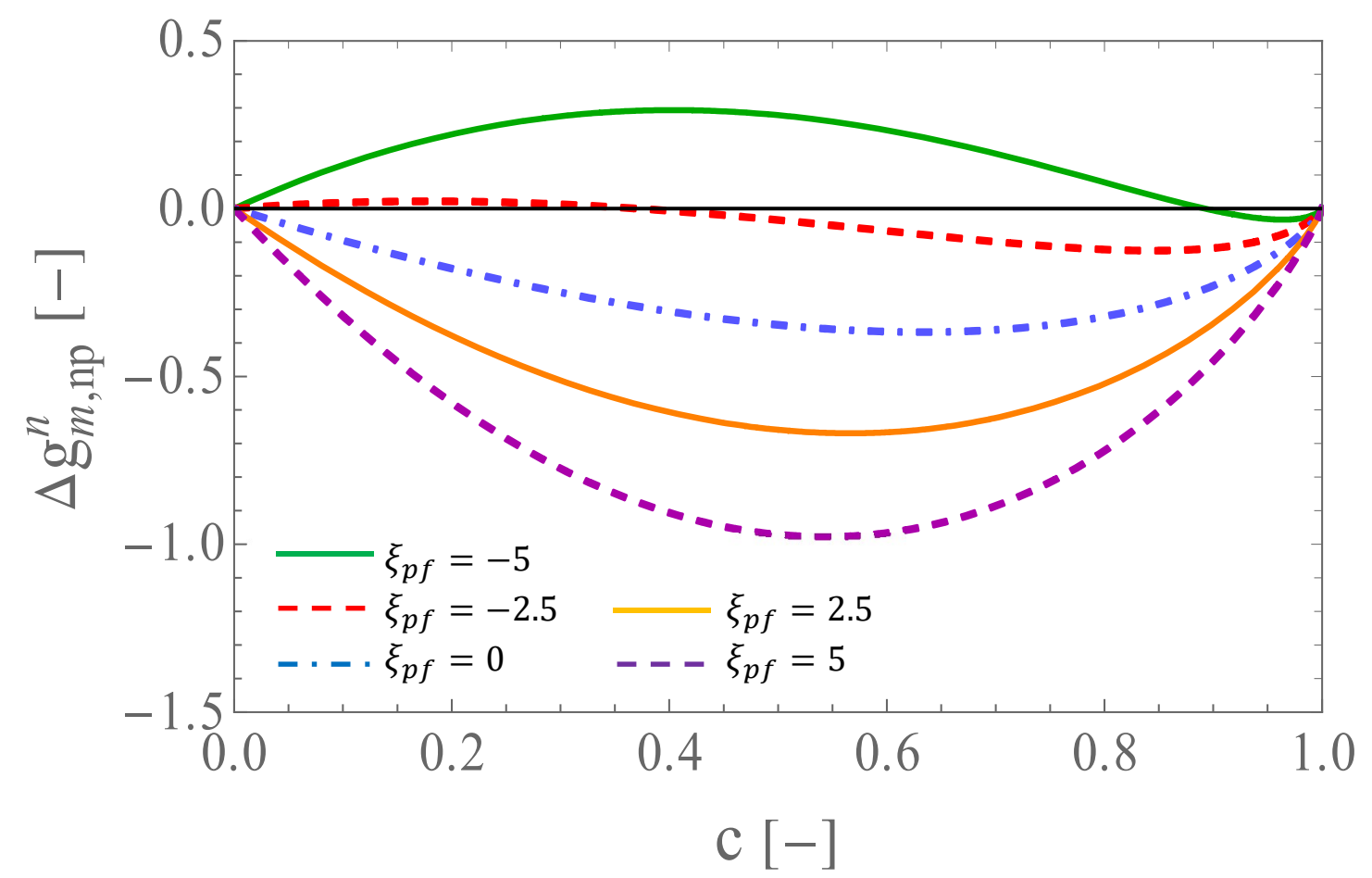

Figure 5: Non-dimensional free energy for regular nanoparticle dispersion $\Delta g_{m, n p}^{n}$ as a function of nanoparticle mass fraction $c$.

A negative value of $\xi_{p f}$ would correspond to a less stable nanofluid that could exhibit a weak interaction between the nanoparticles and the fluid, due to the weak affinity between them. It should be noted that if the negative value of $\xi_{p f}$ is not too large, the nanoparticle dispersion can still be stable due to entropic contribution Theoretically, as long as $\Delta g_{m, n p}<0$, there is a mass fraction for which the nanofluid could be stable. This would correspond to a criterion of

$\xi_{p f}>2 c^{-1} \ln (1-c)$

From Fig. 5, we can see that for the curve corresponding to $\xi_{p f}=-2.5$ a small range of mass fractions would give theoretically a slightly unstable nanofluid. For example, for $c=0.1$, stability is achieved if $\xi_{p f} \gtrsim-2.11$, so that for the curve with $\xi_{p f}=-2.5$, the nanofluid would not be stable.

Finally, from Eq. (27) we can find the proposed chemical potential for nanoparticle dispersion 
$\eta_{m, n p}=\frac{k_{B} T}{\rho_{p} V_{p}}\left(\ln \left(e^{-1}(1-c)^{-1}\right)+\frac{\xi_{p f}}{2}(2 c-1)\right)$

\subsection{Application to mass diffusion of a nanoparticle suspension}

There is a gap in experiments or modelling concerning the chemical potential of nanoparticle dispersion. Therefore, the present model can only be verified in the context of an application. Since mass diffusion is often linked to a chemical potential gradient rather straightforwardly, it would be quite logical to use our model and compare it to modelling and experimental works dealing with mass diffusion. For pedagogical purpose, let us study the simple problem of matter diffusion in a two-component suspension of mass fractions $c_{1}$ and $c_{2}$, the temperature $T$ is assumed to be uniform. Since $c_{1}+c_{2}=1$, it follows that the set of independent variables is given by $c_{1}$, the entropy $s$ per unit mass of the system depends on this mass fraction and one has $s=s\left(c_{1}\right)$ or, in terms of the material time derivative

$\frac{d s}{d t}=\frac{\partial s}{\partial c_{1}} \frac{d c_{1}}{d t}=-\frac{\eta}{T} \frac{d c_{1}}{d t}$,

with $\eta$ designating the difference $\eta_{1}-\eta_{2}$ between the chemical potentials of both constituents. Entropy is also assumed to obey a time evolution equation of the general form

$\rho \frac{d s}{d t}=-\nabla \cdot \boldsymbol{J}^{s}+\sigma^{s}, \quad$ with $\sigma^{s} \geq 0$

$\sigma^{S}$ is the rate of entropy production imposed to be positive definite in virtue of the Second Law of Thermodynamics and $\boldsymbol{J}^{S}$ is the entropy flux. The mass conservation law is given by

$\rho \frac{d c_{1}}{d t}=-\nabla \cdot \boldsymbol{J}_{1}$

where $\boldsymbol{J}_{1}$ is the mass flux of component 1. Comparing (31)-(33) with one another at equilibrium $\left(\sigma^{s} \equiv 0\right)$, we easily find that the equilibrium entropy flux is classically given by

$\boldsymbol{J}^{S}=-\frac{\eta}{T} \boldsymbol{J}_{1}$

At non-equilibrium the entropy production is positive (second law), and we obtain, by filling (33) in (31), (34) in (32) and comparing the two results, the classical expression for the entropy production

$\sigma^{s}=-J_{1} \cdot \frac{\nabla \eta}{T} \geq 0$

The simplest way guaranteeing the positiveness of relation (35) is to assume that there exists a linear relation between the flux $J_{1}$ and its conjugated force represented by $\frac{\nabla \eta}{T}$, which leads to

$\boldsymbol{J}_{f}=\frac{1}{\chi}\left(-\frac{\nabla \eta}{T}\right)$

defining component 1 by the fluid. The symbol $\chi$ can be found by considering that in the case of equilibrium diffusion, Eq. (36) should reduce to Fick's law, $\boldsymbol{J}_{f}=-\rho D \nabla(1-\mathrm{c})$, with $D$ the 
self-diffusion coefficient $[8,29]$. In this case, the chemical potential gradient can be defined as $\nabla \eta=\frac{\partial \eta}{\partial(1-c)} \nabla(1-\mathrm{c})=-\frac{\partial \eta}{\partial \mathrm{c}} \nabla(1-\mathrm{c})$. This leads to

$\chi \equiv \frac{\partial \eta}{\partial(1-c)} \frac{1}{\rho D T}$

For nanoparticle dispersions, we mentioned earlier that in our model we consider that the fluid flows in between the particles, like in a porous-like medium. In such a case, we can say that diffusion happens rather by a non-Fickian local pressure-diffusion mechanism. The chemical potential gradient in such a case should then be written as $\nabla \eta \equiv \frac{\partial \eta}{\partial p} \nabla p$. Such different formulations of the chemical potentials have been proposed and used in the literature [41-45]. At constant temperature and composition, we can deduce from Gibbs equation that $\frac{\partial \eta}{\partial p} \equiv \frac{1}{\rho}$. From Eq. (13), we have for $\varrho \equiv \rho$ that $\nabla p=\nabla p_{f}=\frac{k_{B} T}{V_{f}} \frac{\rho}{\rho_{f}} \nabla(1-\mathrm{c})$ (recall that in our model, for a dispersion, $\nabla p_{p}=0$ ). It is proven experimentally that $\xi_{p f}$ can depend on the concentration [46-48]. We should then write the enthalpic contribution as $-\frac{\xi_{p f}(c)}{2} c(1-c)$. With this in mind and as Eq. (30) has been obtained by deriving Eq. (28) with respect to c, we have to rewrite $\frac{\partial \eta}{\partial(1-c)}=\frac{\partial^{2} \Delta g_{m, n p}}{\partial(1-c)^{2}}=\frac{k_{B} T}{\rho_{p} V_{p}}\left(\frac{1}{1-c}+\Xi_{p f}(c)\right)$, with $\quad \Xi_{p f}(c)=-2 \xi_{p f}(c)+(2-4 c) \partial_{c} \xi_{p f}(c)+$ $c(1-c) \partial_{c c} \xi_{p f}(c)$. Now, recalling that under our model $\rho_{p} V_{p}=\rho_{f} V_{f}$, Eq. (36) becomes

$\boldsymbol{J}_{f}=-\frac{\rho D}{\frac{1}{1-c}+\Xi_{p f}(c)} \nabla(1-c)$,

Comparing this with an effective Fick's law, i.e. $\boldsymbol{J}_{f}=-\rho D_{n p} \nabla(1-\mathrm{c})$ leads to

$D_{n p}=D_{0} \frac{1-c}{1+\Xi_{p f}(c)(1-c)}$

where we rewrite the self-diffusion coefficient as $D \equiv D_{0}$, for conventional reasons. The symbol $\xi_{p f}$ depends on the interaction the dispersing agents cause between the nanoparticles and the fluid. If there are no nanoparticles, one would deduct that the enthalpy of dispersion should vanish. This suggests that, for the present approach, we can say that $\lim _{c \rightarrow 0} \Xi_{p f}=0$, which is a phenomenon supported by the literature for nanoparticle or colloidal suspensions [49-51]. The precise expression for $\xi_{p f}(c)$ is not the subject of this paper, but for the sake of the discussion, we can, motivated by other works [46-48], take a simple linear relation for $\xi_{p f}$ of the type $\xi_{p f}(c)=\xi_{p f, 0}(1+\alpha c)$, with $\alpha=O(1)$. We will take for simplicity $\alpha \equiv 1$ and $\xi_{p f, 0}$ is now given by Eq. (6). Then, we would obtain $\Xi_{p f}=3 \xi_{p f, 0} c$, and we can easily see that $\lim _{c \rightarrow 0} D_{n p}=D_{0}$, which is what would be expected from a nanoparticle suspension or dispersion. In [49], they stated that in the limit of very small volume fractions, the repulsive interactions between colloids (we make an analogy with nanoparticles) are negligible and propose a diffusion coefficient from hydrodynamic predictions of the form $D_{h y}=D_{0}(1-2.1 \varphi)$. The factor -2.1 is a theoretical value obtained by $[52,53]$. If we neglect the interaction forces in Eq. (39), we end up with 
$D_{n p, s}=D_{0}\left(1-\frac{\rho_{p}}{\rho} \varphi\right)$,

which shows a similar behaviour. For appreciation, the hydrodynamic prediction and our model neglecting interaction forces, Eq. (40), are traced in Fig. 6 together with some Molecular Dynamics (MD) data of the diffusion coefficient for a colloid with $\frac{\rho_{p}}{\rho_{f}}=5$ [49,54]. The MD data differ in the mean free paths for the diffusion being a treated subject in that work, but this is not of importance for the present discussion (more information on this can be found in [49]). We can see in Fig. 6 that Eq. (40) actually depicts the general tendency quite well.

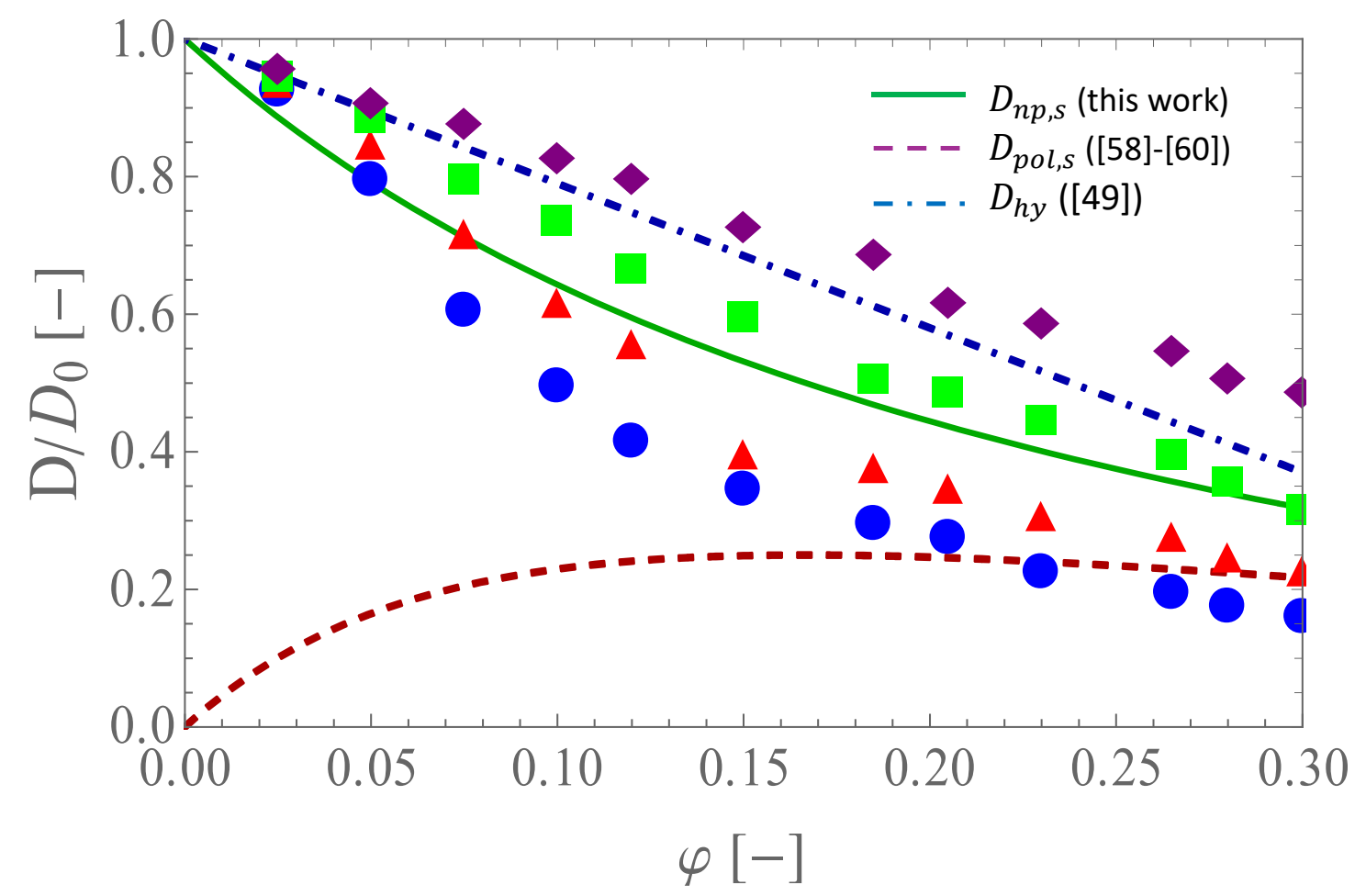

Figure 6: Comparison MD data (markers) from [49] to equations for the diffusion coefficient: $D_{h p, s}, D_{h y}$ and $D_{p o l, s}$.

Let us recall that these results have been obtained by using Eq. (28) and that the same tendency has been observed in other works [55-57]. If we had used Eq. (18), we would have obtained that

$D_{p o l}=D_{0} \frac{c(1-c)}{1+\Xi_{p f}(c) c(1-c)}$

which is not at all suitable for nanoparticle suspensions. Moreover, for Eq. (41) it holds that $\lim _{c \rightarrow 0} D_{e f f, p o l}=0$, which is not valid for nanoparticle suspensions, but rather widely used for polymeric blends [58-60]. Neglecting interaction forces, Eq. (41) becomes

$D_{p o l, s}=D_{0} \frac{\rho_{p}}{\rho} \varphi\left(1-\frac{\rho_{p}}{\rho} \varphi\right)$ 
For comparison, Eq. (42) is plotted in Fig. 6, which clearly shows that Eq. (40) seems to portrait the behaviour of the diffusion coefficient as a function of the volume fraction much better than Eq. (42). Moreover, we can see, recalling that $\rho=\varphi \rho_{p}+(1-\varphi) \rho_{f}$, that for small $\varphi, D_{n p, s}=$ $D_{0}\left(1-\frac{\rho_{p}}{\rho} \varphi\right)=D_{0} \frac{(1-\varphi) \rho_{f}}{\varphi \rho_{p}+(1-\varphi) \rho_{f}} \approx D_{0} \frac{\rho_{f}}{\varphi \rho_{p}+\rho_{f}}=D_{0} \frac{1}{1+\varphi \frac{\rho_{p}}{\rho_{f}}}$ and that for large $\varphi, D_{n p, s}=$ $D_{0}\left(1-\frac{\rho_{p}}{\rho} \varphi\right) \approx D_{0}\left(1-\frac{\varphi \rho_{p}}{\rho_{p}}\right)=D_{0}(1-\varphi)$. This behaviour of a reciprocal dependence on $\varphi$, when small, and a linear dependence on $\varphi$, when large, is also observed in [49,54].

There are works where the behaviour of the diffusion coefficient as a function of the nanoparticle or colloid concentration is different, but these involve often electrostatic or chemical mechanisms that tend to influence the particles' trajectory, e.g. [61]. Incorporating such effects would necessitate the extension of the chemical potential. Moreover, in some studies, adding a soluble substance, such as a solute (and not a particle), to a nanofluid seems to increase the diffusion coefficient, which could be understood by a supplementary entropy increase due the combinatorial mixing of this solute, e.g. [62]. Such contradictory behaviour can be understood by examining Eq. (39) and recalling the example $\Xi_{p f}=3 \xi_{p f, 0} c$ mentioned there. If $\xi_{p f, 0}<0$, the nanoparticles would be repulsive to the base fluid. Looking at Eq. (39), we can easily see that, depending on the magnitude $\left|\xi_{p f, 0}\right|$, the diffusion coefficient will either increase continuously as a function of the nanoparticle mass fraction (remaining in the diluted range) or may go through a maximum before decreasing. If, however, $\xi_{p f, 0}>0$, the nanoparticles would be attractive to the base fluid and Eq. (39) shows that the diffusion coefficient would decrease continuously with the nanoparticle mass fraction. Such behaviours and the concept of nanoparticle-base fluid (or liquid matrix which may be polymeric) repulsion or attraction and its influence on the diffusion coefficient has been observed by molecular dynamics simulations in $[63,64]$. A similar maximum in the diffusion coefficient is also experimentally measured in [65]. Nevertheless, there are quite some experimental data that show a decreasing trend of the diffusion coefficient of nanoparticles or colloids suspended or dispersed in fluids in the same manner as predicted by Eqs. (39)-(40). For illustration, we will compare our model, Eq. (40), with experimental data where particle-fluid interaction is weak or can be neglected. In [66], they measured the diffusion coefficients of silica particles in cycloheptane. Although it concerned two types of hard, spherical silica particles [66], they used an experimental technique (photon correlation spectroscopy) that allowed to measure the diffusion coefficient $D_{n p, s}$ as a function of the overall mass concentration. For our model, we convert the mass concentration to volume fraction, we use the average density of the silica particles they used, i.e. $\rho_{p} \approx 1825 \mathrm{~kg} / \mathrm{m}^{3}$, a cyclohexane density of $\rho_{f}=807 \mathrm{~kg} / \mathrm{m}^{3}$ and a selfdiffusion coefficient $D_{0} \approx 4.5 * 10^{-12} \mathrm{~m}^{2} / \mathrm{s}$ [66]. On a side note, in order to include size effects, Eqs. (31) and (34) can be extended in the same manner as in $[29,67]$. However, the silica particles had sizes of approximately $35 \mathrm{~nm}$, for which size effects could be neglected for diffusion in cycloheptane under their experimental conditions. The results are traced in Fig. 7. 


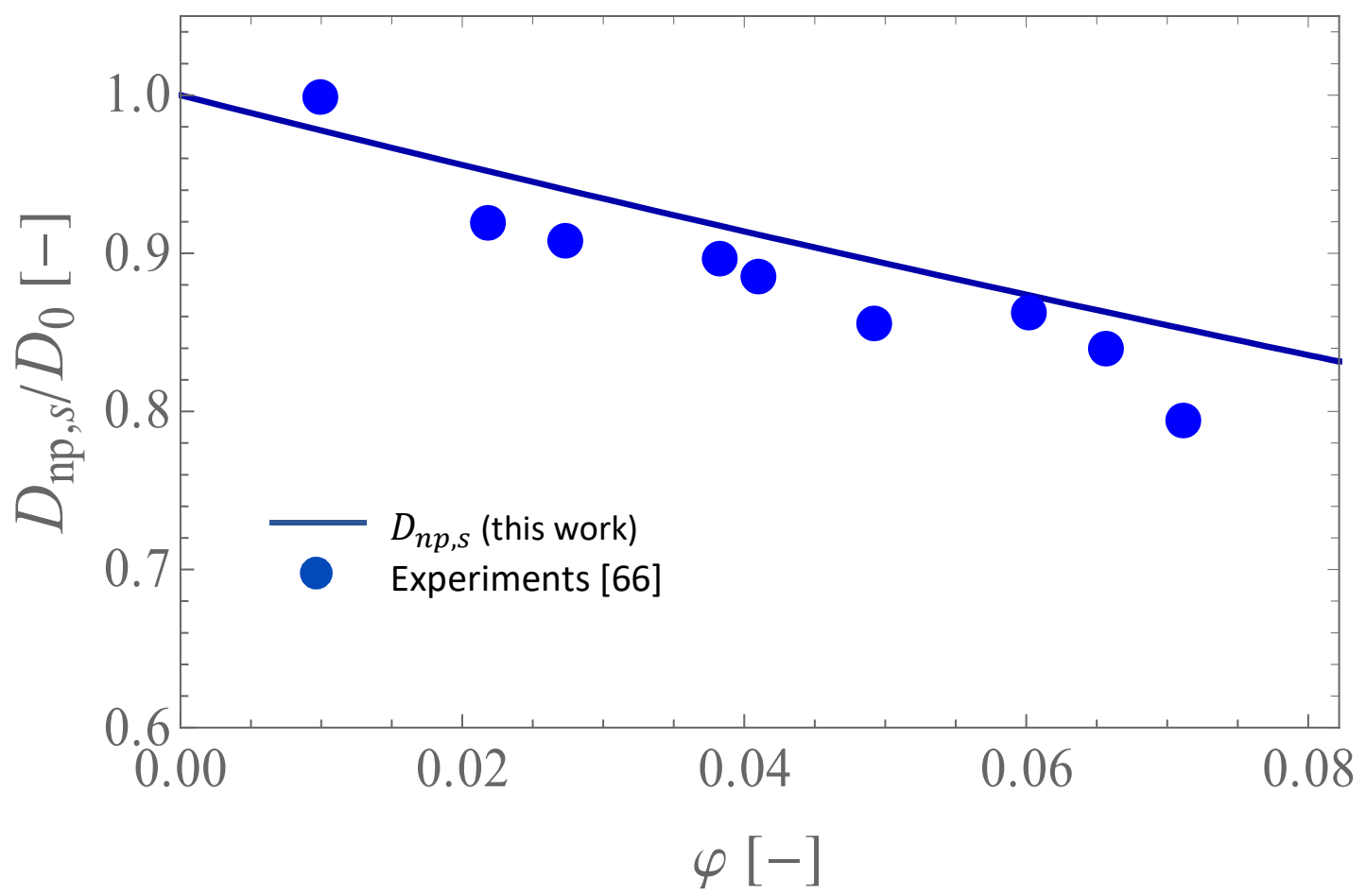

Figure 7: The effective diffusion coefficient $D_{n p, s}$ from Eq. (40) compared to experimental data of silica nanoparticles in cyclohexane [66].

We also compare our model with experiments of nanoparticle dispersion in a weakly entangled polymer blend, where the latter is defined such that $\varepsilon_{p f}=\varepsilon_{p p}=\varepsilon_{f f}$, so that $\xi_{p f}=0$ and we can neglect the enthalpic contribution [68]. The density of the polymer melt, poly-methyl methacrylate (PMMA), is not given there explicitly, but from information on the interaction energy $\varepsilon_{f f}$, the inter-monomer interaction distance $\sigma_{m}$, the operating pressure and temperature [68] as well as the glass temperature [69,70], we can estimate by extrapolation [69] a density of $\rho_{f} \approx 880 \mathrm{~kg} / \mathrm{m}^{3}$. At such temperatures and pressure, the density of silica is not much changed [71] and an approximate value of $\rho_{p} \approx 2400 \mathrm{~kg} / \mathrm{m}^{3}$ can be found [71,72]. The results are shown in Fig. 8. 


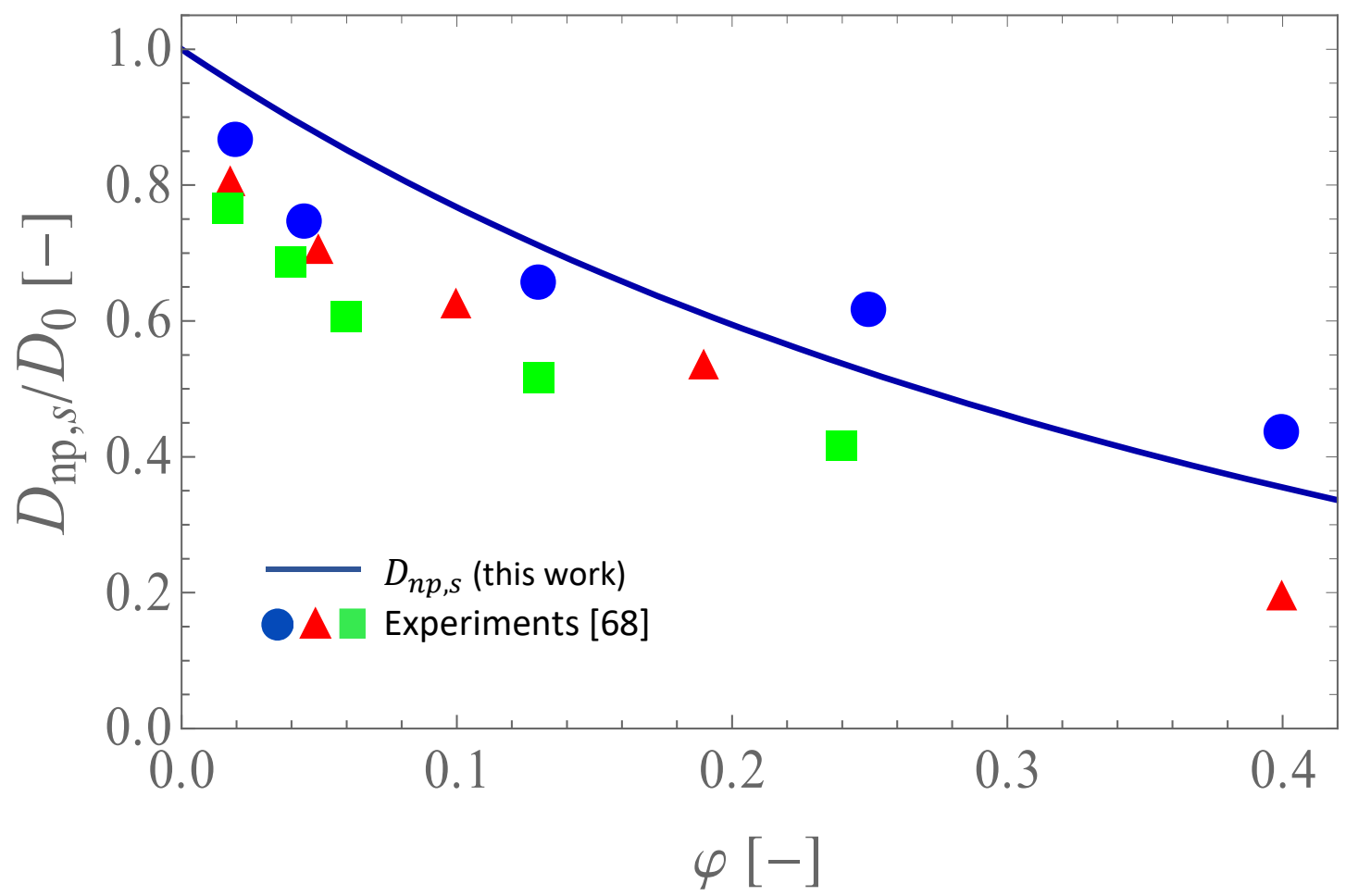

Figure 8: The effective diffusion coefficient $D_{n p, s}$ from Eq. (40) compared to experimental data of silica nanoparticles in PMMA melts [68].

The results in Figs. 7 and 8 show a good comparison with the experimental data, which suggests a correct representation of the chemical potential of dispersion proposed in this work. Moreover, it also suggests the density-dependence of the chemical potential. Still other works show similar behaviour of the diffusion coefficient, e.g. nanoparticle diffusion in electrolytic solutions for batteries [73,74], protein diffusion in membranes [75], nanoparticle diffusion in volatile liquids [76] and aqueous cellulose nanofibers diffusion [77]. This substantiates the use of the chemical potential for nanoparticle/colloidal suspensions/dispersions proposed in this work.

\section{Conclusions}

For nanoparticle suspensions, a new analytical model is proposed for the chemical potential of nanoparticle dispersions that could be straightforwardly used for material properties. We have discussed that it is conceptually not reasonable to consider nanofluids (as they are used for practical applications) as mixtures in the sense of binary solutions on a molecular level. By means of thermodynamic considerations and proposing a porous-like model for fluid diffusion in between nanoparticles, we obtained an expression for the chemical potential for nanoparticle dispersion. From this expression, the same expression for the osmotic pressure of a dispersion was obtained as by a kinetic study [36]. Moreover, by a series of simplifications, the Van't Hoff equation was readily deduced. In order to illustrate the importance of a correct chemical potential, we used the one in this paper and the standard one to deduce the diffusion coefficient of nanoparticle diffusion in a fluid as a function of the mass fraction. There are, of course, other parameters on which the diffusion coefficient depends, such as particle size, surface chemistry, fluid composition, electrostatic effects, active particles. For purposes related to the chemical potential, we rather deal here with entropic dispersing effect (having also discussed the 
enthalpic one). The proposed chemical potential is verified further by developing a mass diffusion coefficient using both approaches, the standard chemical potential of mixing and that of dispersion. The behaviour of the diffusion coefficient is also explained in [78] by a meanfield hydrodynamic theory in porous medium. Interestingly, this seems to coincide with the porous-like model we used in our work, which lead finally to expression Eq. (28). The comparison with molecular dynamics and experimental data has shown that the use of the standard formulation for the free energy of mixing is not suitable for nanoparticle suspensions/dispersions. The chemical potential we proposed describes, however, correctly the behaviour of nanoparticle diffusion in fluids. The reason for this is explained by the present model itself. It appears from the model that, such as in a porous medium, it is the fluid that diffuses through the nanoparticles, which governs the mechanism behind the chemical potential of dispersion. Moreover, the density-dependence of the chemical potential of dispersion is hereby implied as well. It should be mentioned that, if the base fluids are polymeric of nature in a way that nanoparticles could alter their configuration either chemically or physically, other effects come into play and the model should be extended. It could be argued that the application of our model could also be extended to emulsions and dispersions of materials that are not considered to be a mixture or even to solutions of which the solute is a solid [79]. Finally, the interchange energy $\Delta w$, discussed in relation with the particle-matrix interaction, can be linked to a nano-layer of dense structured fluid around the nanoparticles [80,81]. This could be an interesting alternative to using interaction energies $\varepsilon_{i j}$, since nano-layer thicknesses are sometimes more readily available and can often be taken to be typically of the size $O(1) \mathrm{nm}$ $[80,81]$. This work shows that the present expression for the chemical potential, resulting from a porous-medium-like behaviour, gives quite good results for nanofluid dispersions and suspensions and could be an incentive for further development.

\section{Acknowledgements}

We acknowledge financial support from BelSPo Prodex programme and ESA MAP Evaporation programme.

\section{References}

[1] P.J. Flory, Thermodynamics of High Polymer Solutions, Journal of Chemical Physics (1941) 9, 660-661.

[2] P.J. Flory, Thermodynamics of High Polymer Solutions, Journal of Chemical Physics (1942) 10, 51-61.

[3] M.L. Huggins, Solutions of Long Chain Compounds, Journal of Chemical Physics (1941) 9, 440-441.

[4] J. Wilmers, S. Bargmann, A continuum mechanical model for the description of solvent induced swelling in polymeric glasses: thermomechanics coupled with diffusion, European Journal of Mechanics A/Solids (2015), 53, $10-18$.

[5] J.S. Higgins, J.E.G. Lipson, R.P. White, A simple approach to polymer mixture miscibility, Phil. Trans. R. Soc. A (2010) 368, 1009-1025.

[6] P. Hohenberg, A. Krekhov, An introduction to the Ginzburg-Landau theory of phase transitions and nonequilibrium patterns (2014), Physics Reports 572.

[7] D.J. Kozuch, W. Zhang, S.T. Milner, Predicting the Flory-Huggins $\chi$ parameter

for polymers with stiffness mismatch from molecular dynamics simulations, Polymers (2016), 8, 241.

[8] P. Galenko, D. Jou, Kinetic contribution to the fast spinodal decomposition controlled by diffusion, Physica A 388 (2009) 3113-3123.

[9] M.E. Mackay, A. Tuteja, P.M. Duxbury, C.J. Hawker, B. van Horn, Z. Guan, G. Chen, R.S. Krishnan, General strategies for nanoparticle dispersion, Science (2006), 311, 1740-1743. 
[10] J. van Rijssel, B.H. Erné, J.D. Meeldijk, M. Casavola, D. Vanmaekelbergh, A. Meijerink, A.P. Philipse, Enthalpy and entropy of nanoparticle association from temperature-dependent cryo-TEM, Phys. Chem. Chem. Phys. (2011), 13, 12770-12774.

[11] S.U.S. Choi, J.A. Eastman, Enhancing thermal conductivity of fluids with nanoparticles, in: Conference: 1995 International Mechanical Engineering Congress and Exhibition, ASME, 1995, pp. 99-105.

[12] F. Yu, Y. Chen, X. Liang, J. Xu, C. Lee, Q. Liang, P. Tao, T. Deng, Dispersion stability of thermal nanofluids, Progress in Natural Science: Materials International 27 (2017) 531-542.

[13] H. Machrafi, An extended thermodynamic model for size-dependent thermoelectric properties at nanometric scales: Application to nanofilms, nanocomposites and thin nanocomposite films, Appl. Math. Mod. (2016), 40, 2143-2160.

[14] H. Machrafi, Enhancement of a photovoltaic cell performance by a coupled cooled nanocomposite thermoelectric hybrid system, using extended thermodynamics, Current Appl. Phys. (2017), 17, 890-911.

[15] J. Taha-Tijerina, T.N. Narayanan, G. Gao, M. Rohde, D.A. Tsentalovich, M. Pasquali, P.M. Ajayan, Electrically Insulating Thermal Nano-Oils Using 2D Fillers, ACS Nano 6 (2012) 1214-1220.

[16] A. Cardellini, M. Fasano, M.B. Bigdeli, E. Chiavazzo, P. Asinari, Thermal transport phenomena in nanoparticle suspensions, J. Phys. Condens. Matter 28 (2016) 483003.

[17] Y. Lin, S. Sun, Q. Zhang, H. Shen, Q. Shao, L. Wang, W. Jiang, W. Jiang, Preparation of $\mathrm{AgNPs}_{\mathrm{S}} / \mathrm{Ca}_{3} \mathrm{Co}_{4} \mathrm{O}_{9}$ nanocomposites with enhanced thermoelectric performance, Materials Today Communications (2016), 6, 44-49.

[18] H. Machrafi, G. Lebon, C.S. Iorio, Effect of volume-fraction dependent agglomeration of nanoparticles on the thermal conductivity of nanocomposites: Applications to epoxy resins, filled by $\mathrm{SiO}_{2}, \mathrm{AlN}$ and $\mathrm{MgO}$ nanoparticles, Composites Science and Technology (2016), 130, 78-87.

[19] Z. Hai, M.K. Akbari, Z. Wei, C. Xue, H. Xu, J. Hu, L. Hyde, S. Zhuiykov, $\mathrm{TiO}_{2}$ nanoparticles-functionalized two-dimensional $\mathrm{WO}_{3}$ for high-performance supercapacitors developed by facile two-step ALD process, Materials Today Communications (2017), 12, 55-62.

[20] R. Ahmad, O.S. Wolfbeis, Y.B. Hahn, H.N. Alshareef, L. Torsi, K.N. Salama, Deposition of nanomaterials: A crucial step in biosensor fabrication, Materials Today Communications (2018), 17, 289-321.

[21] R. Saidur, K.Y. Leong, H. Mohammad, A review on applications and challenges of nanofluids, Renew. Sustain. Energy Rev. 15 (2011), 1646-1668.

[22] J.M.M. de la Hoz, R.C. Tovar, P.B. Balbuena, Size effect on the stability of $\mathrm{Cu}-\mathrm{Ag}$ nanoalloys, Molecular Simulation (2009), 35, 785-794.

[23] S. Ohja, A. Dang, C.M. Hui, C. Mahoney, K. Matyjaszewski, M.R. Bockstaller, Strategies for the synthesis of thermoplastic polymer nanocomposite materials with high inorganic filling fraction, Langmuir (2013), 29, 89898996.

[24] J.L. Dominguez-Juarez, S. Vallone, A. Lempel, M. Moocarme, J. Oh, H.D. Gafney, L.T. Vuong, Influence of solvent polarity on light-induced thermal cycles in plasmonic nanofluids (2015), Optica 2, 447-453.

[25] C.S. Lovell, K.E. Wise, J.W. Kim, P.T. Lillehei, J.S. Harrison, C. Park, Thermodynamic approach to enhanced dispersion and physical properties in a carbon nanotube/polypeptide nanocomposite, Polymer 50 (2009) 19251932.

[26] I. Borukhov, L. Leibler, Enthalpic stabilization of brush-coated particles in a polymer Melt, Macromolecules (2002), 35, 5171-5182.

[27] B.D. Anderson, Predicting solubility/miscibility in amorphous dispersions: it is time to move beyond regular solution theories, Journal of Pharmaceutical Sciences (2018), 107, 24-33.

[28] W.C. Williams, Experimental and theoretical investigation of transport phenomena in nanoparticle colloids (Nanofluids), PhD thesis MIT, 2006.

[29] H. Machrafi, G.Lebon, Fluid flow through porous and nanoporous media within the prism of extended thermodynamics: emphasis on the notion of permeability, Microfluidics and Nanofluidics, 22:65 (2018)1-12.

[30] S. Ichihara, Glass transition temperature of homogeneous blends and copolymers, Polymer Journal (2000), 32, 823-827.

[31] A.T. Slark, Application of the Kwei equation to the glass transition of dye solute-polymer blends, Polymer (1999), 40, 1935-1941.

[32] G. Nicolis, Y. de Decker, Stochastic thermodynamics of Brownian motion, Entropy (2017), $19,434$.

[33] K.R. Sharma, Nanostructuring of nanorobots for use in nanomedicine, International Journal of Engineering and Technology (2012), 2, 116-134.

[34] T.K. Kwei, E.M. Pearce, J.R. Pennacchia, M. Charton, Correlation between the glass transition temperatures of polymer mixtures and intermolecular force parameters, Macromolecules (1987), 20, 1174-1176. 
[35] T.K. Kwei, The effect of hydrogen bonding on the glass transition temperatures of polymer mixtures, Macromolecules (1984), 22, 307-313.

[36] P.H. Nelson, Osmosis and thermodynamics explained by solute blocking, Eur Biophys J. (2017), 46, 59-64.

[37] T.F. Tadros, Interfacial Phenomena and Colloid Stability, De Gruyter, 2015.

[38] A. Miermont, F. Waharte, S. Hu, M.N. McClean, S. Bottani, S. Léon, P. Hersen, Severe osmotic compression triggers a slowdown of intracellular signaling, which can be explained by molecular crowding, PNAS (2013), 110, 5725-5730.

[39] A.A. Zavitsas, Properties of water solutions of electrolytes and nonelectrolytes, J. Phys. Chem. B (2001), 105, 7805-7817.

[40] J. Rodenburg, M. Dijkstra, R. van Roij, Van't Hoff's law for active suspensions: the role of the solvent chemical potential, Soft Matter (2017), 13, 8957-8963.

[41] H.M. Vollebregt, R.G.M. van der Sman, R.M. Boom, Suspension flow modelling in particle migration and microfiltration, Soft Matter (2010), 6, 6052-6064.

[42] Y. A. Buyevich, Particle distribution in suspension shear flow, Chem. Eng. Sci., 1996, 51(4), 635-647.

[43] I. C. Carpen and J. F. Brady, Gravitational instability in suspension flow., J. Fluid Mech., 2002, 472, 201210.

[44] A. Fang, A.A. Mammoli, J.F. Brady, M.S. Ingber, L.A. Mondy, A.L. Graham, Flow-aligned tensor models for suspension flows, Int. J. Multiphase Flow (2002), 28, 137-166.

[45] D. Leighton, A. Acrivos, Viscous resuspension, Chem. Eng. Sci. (1986), 41, 1377-1384.

[46] A.A. Teran. N.P. Balsara, Thermodynamics of Block Copolymers with and without Salt, J. Phys. Chem. B (2014), 118, 4-17.

[47] K.S. Schweizer, J.G. Curro, Analytic reference interaction site model-mean spherical approximation theory of flexible polymer blends: Effects of spatial and fractal dimensions, J. Chem. Phys. (1991), 94, 3986-4000. [48] K. Kamide, S. Matsuda, M. Saito, Evaluation of concentration dependence of $\chi$-parameter, flory temperature and entropy parameter for polymer-solvent system from their critical solution temperature and concentration data.

[49] M. Ripoll, K. Mussawisade, R.G. Winkler, G. Gompper, Dynamic regimes of fluids simulated by multiparticle-collision dynamics, Phys. Rev. E (2005) 72, 016701.

[50] T. Burghelea, V. Bertola, Transport Phenomena in Viscoelastic Fluids, Springer, 2019

[51] R.R. Huilgol, N. Phan-Thien, Fluid Mechanics of ViscoElasticity, Elsevier, 1997.

[52] G.K. Batchelor, Brownian diffusion of particles with hydrodynamic interaction, Journal of Fluid Mechanics (1976), 74, 1.

[53] W.B. Russel, D.A. Saville, W.R. Schowalter, Colloidal Dispersions (Cambridge University Press, 2001). [54] J.P. Boon, S. Yip, Molecular Hydrodynamics, (McGraw-Hill, New York, 1980).

[55] H.D. Newman, A. Yethiraj, Clusters in sedimentation equilibrium for an experimental hard-sphere-plusdipolar Brownian colloidal system, Scientific Reports (2015), 5, 13572.

[56] M.H. Blees, J.M. Geurts, J.C. Leyte, Self-Diffusion of Charged Polybutadiene Latex Particles in Water Measured by Pulsed Field Gradient NMR, Langmuir (1996), 12, 1947-1957

[57] C.G. de Kruif, J.W. Jansen, A. Vrij, In S.A. Safran, N.A. Clark, (eds.) Physics of Complex and Supramolecular Fluids (Wiley, New York, 1987).

[58] K.Y. Ozawa, T. Okuzono, M. Doi, Diffusion process during drying to cause the skin formation in polymer solutions. Jpn. J. Appl. Phys. (2006) 45, 8817.

[59] G.A. Zoumpouli, S.G Yiantsios, Hydrodynamic effects on phase separation morphologies in evaporating thin films of polymer solutions, Physics of fluids (2016), 28, 082108.

[60] E.B. Nauman, D.Q. He, Nonlinear diffusion and phase separation, Chemical Engineering Science 56 (2001) 1999-2018.

[61] J.P. Holmberg, Z. Abbas, E. Ahlberg, M. Hassellöv, J. Bergenholtz, Nonlinear concentration dependence of the collective diffusion coefficient of $\mathrm{TiO}_{2}$ nanoparticle dispersions, J. Phys. Chem. C (2011), 115, 13609-13616. [62] X. Fang, Y. Xuan, Q. Li, Experimental investigation on enhanced mass transfer in nanofluids, Applied Physics Letters (2009), 95, 203108.

[63] T. Desai, P. Keblinski, S.K. Kumar, Molecular dynamics simulations of polymer transport in nanocomposites, The Journal of Chemical Physics, (2005), 122, 134910.

[64] G. D. Smith, D. Bedrov, L. Li, O. Byutner, A molecular dynamics simulation study of the viscoelastic properties of polymer nanocomposites, J. Chem. Phys. (2002), 117, 9478.

[65] A. Mouhli, H. Ayeb, T. Othman, J. Fresnais, V. Dupuis, I.R. Nemitz, J.S. Pendery, C. Rosenblatt, O. 
Sandre, E. Lacaze, Influence of a dispersion of magnetic and nonmagnetic nanoparticles on the magnetic Fredericksz transition of the liquid crystal 5CB, Physical Review E (2017), 96, 012706.

[66] M.M. Kops-Werkhoven, C. Pathmamanoharan, A. Vrij, H.M. Fijnaut, Concentration dependence of the self-diffusion coefficient of hard, spherical particles measured with photon correlation spectroscopy, J. Chem. Phys. 77 (1982), 5913-5922.

[67] D. Jou, J. Casas-Vazquez, M. Criado-Sancho, Thermodynamics of fluids under flow, Springer, 2011. [68] A. Karatrantos, R.J. Composto, K.I. Winey, N. Clarke, Polymer and spherical nanoparticle diffusion in nanocomposite, J. Chem. Phys. (2017), 146, 203331.

[69] M. Schmidt, F.H.J. Maurer, Pressure-volume-temperature properties and free volume parameters of PEO/PMMA blends, Journal of Polymer Science: Part B: Polymer Physics (1998), 36, 1061-1080.

[70] G. Wen, Z. Sun, T. Shi, J. Yang, W. Jiang, L. An, B. Li, Thermodynamics of PMMA/SAN blends: application of the Sanchez-Lacombe lattice fluid theory, Macromolecule (2001), 34, 6291-6296.

[71] I. Iosilevskiy, V. Gryaznov, A. Solovev, Properties of high-temperature phase diagram and critical point parameters in silica, High Temp.-High Press. (2014), 43, 227-241.

[72] I.V. Schweigert, K.E.J. Lehtinen, M.J. Carrier, M.R. Zachariah, Structure and properties of silica nanoclusters at high temperatures, Physcial Review B (2002), 65, 235410.

[73] K. Higa, S.L. Wu, D.Y. Parkinson, Y. Fu, S. Ferreira, V. Battaglia, V. Srinivasan, Comparing macroscale and microscale simulations of porous battery electrodes, Journal of the Electrochemical Society (2017), 164, E3473-3488.

[74] S.G. Stewart, J. Newman, The Use of UV/vis Absorption to Measure Diffusion Coefficients in LiPF6 Electrolytic Solutions, J. Electrochem. Soc., 155(1), F13-F16 (2008).

[75] P.F. Almeida, W.L.C. Vaz, Lateral diffusion in membranes, Handbook of Biological Physics (1995), 1, 305357.

[76] D.K. Rathore, S. Kumar, Experimental investigations on diffusion of nano-additive volatile liquids in gas, Int. J. for Research in Appl. Sc. And Engineering Technolgy (2018), 6, 1450-1456.

[77] R. Motohashi, I. Hanasaki, Characterization of aqueous cellulose nanofiber dispersions from microscopy movie data of Brownian particles by trajectory analysis, Nanoscale Advances (2019), 1, 421-429.

[78] K.K. Senanayake, N. Shokeen, E.A. Fakhrabadi, M.W. Liberatore, A. Mukhopadhyay, Diffusion of nanoparticles within a semidilute polyelectrolyte solution, Soft Matter (2019), 15, 7616-7622.

[79] H.J. Price, J. Mattsson, B.J. Murray, Sucrose diffusion in aqueous solution, Phys Chem Chem Phys (2016), 18, 19207-19216.

[80] L. Wang, J. Fan, Toward nanofluids of ultra-high thermal conductivity, Nanoscale Research Letters (2011), $6,153$.

[81] K. Voïtchovsky, J.J. Kuna, S.A. Contera, E. Tosatti, F. Stellacci, Direct mapping of the solid-liquid adhesion energy with subnanometre resolution, Nature Nanotechnology (2010), 5, 401-405. 